\title{
CSTI Promoted Gastric Cancer Migration and Invasion Through Activating Wnt Pathway
}

This article was published in the following Dove Press journal:

Cancer Management and Research

\section{Si Chen* \\ Yingling Liu* \\ Kaiguang Zhang \\ Lele Chen}

Department of Gastroenterology, The First Affiliated Hospital of USTC, Division of Life Sciences and Medicine, University of Science and Technology of China, Hefei, 23000I, Anhui, People's Republic of China

*These authors contributed equally to this work
Correspondence: Si Chen

Department of Gastroenterology, The First Affiliated Hospital of USTC, Division of Life Sciences and Medicine, University of Science and Technology of China, \#17 Lvjiang Road, Hefei, 23000I, Anhui,

People's Republic of China

Email css81729@sina.com
Introduction: Gastric cancer is one of the main reasons of cancer-induced death, exploring the molecular mechanisms of gastric cancer progression is critical for gastric cancer therapy. Here, we studied the role of cysteine protease inhibitor CST1 in gastric cancer progression. Methods: Matrigel-coated or -uncoated transwell assay was used to determine the effect of CST1 on gastric cancer invasion and migration, luciferase reporter system was used to determine the effect of CST1 on Wnt pathway activity.

Results: CST1 had high expression levels in gastric cancer tissues and cells, patients who had high CST1 expression had poor outcome. Overexpression of CST1 increased gastric cancer migration and invasion, while knockdown of CST1 suppressed gastric cancer migration invasion. Mechanism analysis showed CST1 promoted WNT signaling pathway activity, promoted the nuclear translocation of $\beta$-catenin and the expression of Wnt signaling targets. Inhibition of Wnt pathway in CST1 overexpression cells inhibited migration and invasion, suggesting CST1 promoted gastric cancer cell migration and invasion through activating the Wnt pathway.

Conclusion: In summary, we found CST1 promoted gastric cancer migration and invasion through activating Wnt signaling, providing a novel target for gastric cancer therapy.

Keywords: CST1, gastric cancer, invasion, migration, Wnt pathway

\section{Introduction}

Gastric cancer is the third leading cause of cancer deaths in the world, with East Asia accounting for more than half of these cases. However, even with therapeutic method improvements, the clinical outcome for advanced gastric cancer patients is still poor, ${ }^{1,2}$ so it is urgent to understand the regulatory mechanism of gastric cancer initiation and progression.

Cysteine proteases play a critical role in development of cancer through degradation of extracellular matrix components and modulation of immune responses. Cystatin superfamily can inhibit the proteolytic activities of cysteine proteases. CST1 belongs to the cystatin superfamily and is a cysteine protease inhibitor. ${ }^{3-5}$ CST1 has been reported to regulate tumor progression, for example, CST1 promotes pancreatic cancer growth. ${ }^{6}$ CST1 is a new biomarker for colorectal cancer, it promotes colorectal cancer proliferation, let-7 is a suppressor for many tumors, CST1 is a target of let-7, let-7 inhibits colorectal proliferation through targeting CST1. ${ }^{7,8}$ In non-small cell lung cancer (NSCLC) high CST1 is related with high recurrence and metastasis and low survival rate, and is an independent prognostic factor for NSCLS patients. ${ }^{9}$ CST1 also is a poor prognosis factor for breast cancer patients as it promotes breast cancer proliferation. ${ }^{10} \mathrm{CST} 1$ promotes gastric cancer 
proliferation, ${ }^{11}$ but its role in gastric cancer migration and invasion has not been studied. Here, we studied the effect of CST1 overexpression or knockdown on gastric cancer migration and invasion and found CST1 promoted gastric cancer migration and invasion through activating Wnt signaling.

\section{Materials and Methods Cell Culture and Specimens}

Gastric cancer cell lines SNU-16, SNU-6, SNU-1, NCIN87, and KATO II were purchased from ATCC and maintained in RPMI-1640 (Hyclone) supplemented with 10\% FBS (Hyclone). Immortalized normal gastric epithelial cells were also maintained in RPMI-1640 (Hyclone) supplemented with 10\% FBS (Hyclone). Eight pairs of gastric cancer tissues (T) and adjacent normal gastric tissues (ANT) were collected during surgical procedures from patients with HCC according to the protocol approved by the institutional review board of the Department of Gastroenterology, The First Affiliated Hospital of University of Science and Technology of the People's Republic of China. All patients provided written, informed consent for participation in the study and provision of tumor samples.

\section{Q-PCR and Western Blot}

Total RNA was isolated from cells using the Total RNA Extraction Reagent (Vazyme) and reversed-transcribed into cDNA using HiScript III SuperMix for qPCR (+gRNA wiper) (Vazyme). mRNA expression was determined by Q-PCR using the AceQ Universal SYBR qPCR Master Mix using a CFX-96 Touch Real-Time PCR Detection System (Bio-Rad) was performed according to a standard method. ${ }^{12}$ The following primary antibodies were used: anti-CST1 (ab68329, Abcam), anti- $\beta$-catenin (8480, CST), anti-p84 (ab487, Abcam), anti-E-Cadherin (14472, CST), $\alpha$-Catenin (2131, CST), Fibronectin (26836, CST), Vimentin (5741, CST) and anti-GAPDH (5174, CST). Nuclear extracts were prepared using a Nuclear Extraction Kit (Keygen Biotech).

\section{Vector Construction and Compounds}

The CDS sequence of CST1 was cloned to lentivirus vector pSin-EF1-Puro vector, two shRNA sequences of CST1 were cloned to lentivirus vector PLKO.1-Puro vector, the target sequences for CST1 were shown as following, shRNA\#1, 5'CGAGTATAACAAGGCCACCAA3', and shRNA\#2, 5' GAAGAAACAGTTGTGCTCTTT3'. Wnt pathway inhibitor iCRT3 was purchased from Selleck.

\section{Matrigel-Coated or -Uncoated Transwell Assay}

Transwell chambers assay without or with coated Matrigel (Corning) was used to analyze cell migration and invasion. ${ }^{13}$ The lower chamber of the transwell device using 500 ul DMEM supplemented with 10\% FBS, after 24 hours of incubation, cells invading into the bottom side of the inserts were fixed using $4 \%$ paraformaldehyde, stained using $1 \%$ purple crystal, photographed, and quantified by counting them in five random high-power fields.

\section{Luciferase Reporter Assay}

A $\beta$-catenin reporter system named M50 Super $8 \times$ TOP Flash (\#12456) was purchased from addgene and transfected into cells using Lipofectamine 3000 (Thermo), according to the manufacturer's instructions. Luciferase activity was analyzed using Dual Luciferase Reporter Assay Kit (Vazyme).

\section{Statistical Analysis}

All statistical analyses were carried out using SPSS 19.0 and GraphPad. Survival curves were analyzed using the Kaplan-Meier method, and the survival differences were compared using the Log rank test, the data was downloaded from the TCGA dataset. Continuous data were compared using Student's two-tailed $t$ test. $P<0.05$ was considered statistically significant.

\section{Results}

\section{CSTI was Upregulated in Gastric Cancer Tissues and Positively Correlated with Poor Clinic Outcome}

To explore the role of CST1 in gastric cancer progression, we firstly determined CST1 expression in gastric cancer tissues. CST1 was significantly upregulated in gastric cancer tissues through analyzing gene expression profiles of gastric cancer tissues and normal gastric epithelial tissues in the TCGA dataset (Figure 1A). Western blot analysis also showed CST1 expression was higher in gastric cancer tissues than adjacent normal gastric epithelial tissues (Figure 1B). Survival analysis showed patients with high CST1 expression had 
A

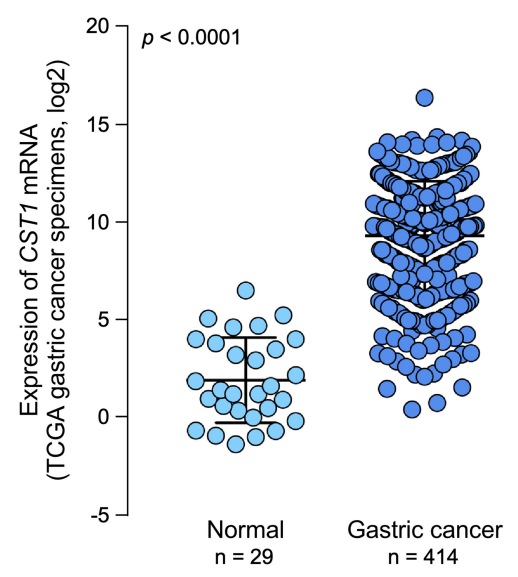

B

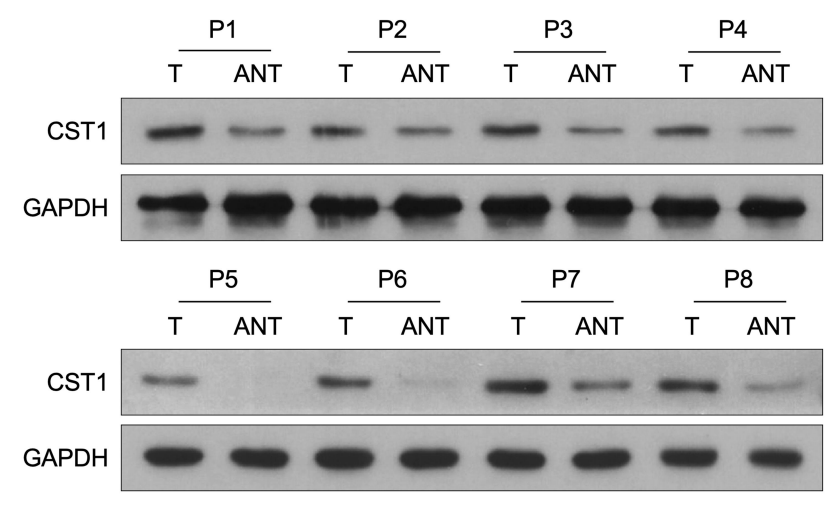

C
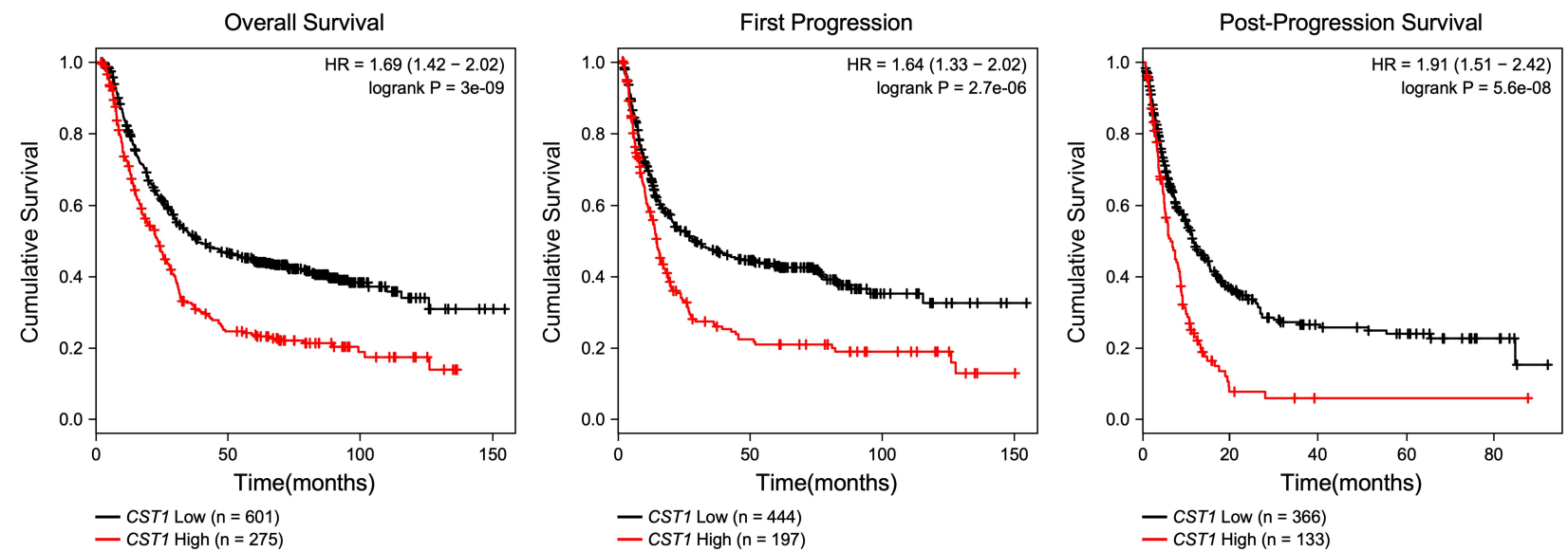

Figure I CSTI is upregulated in gastric cancer tissues and associated with poor outcome. (A) CSTI was significantly upregulated in gastric cancer tissues compared to normal gastric tissues, data were downloaded from the TCGA dataset. (B) Western blot determined CSTI expression in gastric cancer tissues and adjacent normal gastric tissues, GAPDH was used as the loading control. (C) Survival curves showed patients with high CSTI expression had poor outcome.

shorter overall survival, and shorter first progression and post progression survival times than those with low CST expression, data was downloaded from the TCGA dataset (Figure 1C). These results showed CST1 was upregulated in gastric cancer tissues and associated with poor outcome.

\section{CSTI Promoted Gastric Cancer Migration and Invasion}

To study the function of CST1 in gastric cancer development, CST1 expression in gastric cancer cells and normal gastric cells were determined. Western blot suggested CST1 was higher in gastric cancer cells than in normal gastric cells, and it had higher expression in gastric cancer cells NCI-N87 and KATO II, and lower expression in gastric cancer cells SNU-16, SNU-5 and SNU-1
(Figure 2A). We overexpressed CST1 in SNU-5 and knocked down CST1 in NCI-N87 (Figure 2B). Matrigeluncoated transwell assay showed CST1 overexpression significantly increased the cell migration ability compared to vector control, while CST1 knockdown significantly reduced the cell migration ability compared to scramble control (Figure 2C). Matrigel-coated transwell assay showed that CST1 overexpression significantly increased invasion ability compared to vector control, while CST1 knockdown significantly reduced invasion ability compared to scramble control (Figure 2D). In 3D culture, cells with CST1 overexpression grew into more structurally well-organized spheres with invasive projections compared to control cells, while cells with CST1 knockdown grew into structurally well-organized spheres without invasive projections compared to scramble control cells (Figure 2E). We also determined whether CST1 
A

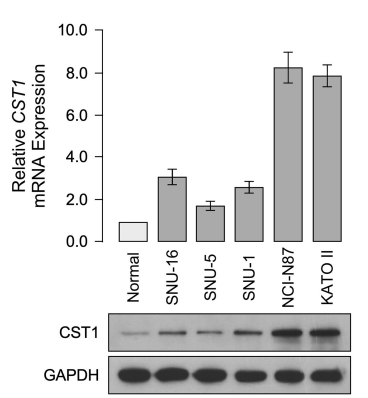

D

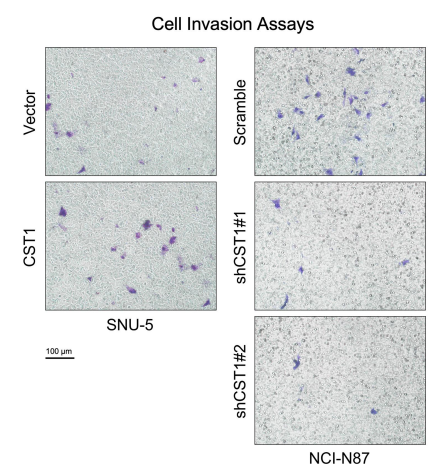

B

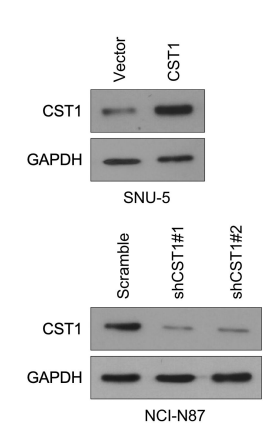

E
C

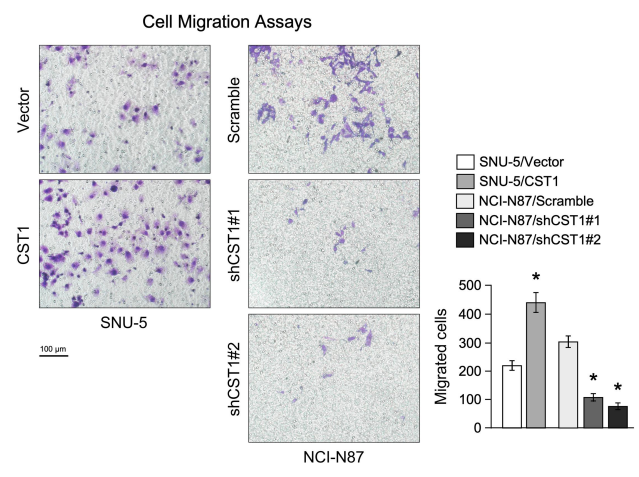

F

Figure 2 CSTI promotes gastric cancer cell migration and invasion. (A) Q-PCR and Western blot determined CSTI expression in normal gastric cell and gastric cancer cells. (B) Western blot determined the effect of CSTI overexpression and CSTI knockdown, GAPDH was used as the loading control. (C) Transwell chambers assay without Matrigel showed CSTI overexpression promoted cellular migration, while CSTI knockdown inhibited cellular migration. (D) Transwell chambers assay with Matrigel showed CSTI overexpression promoted cellular invasion, while CSTI knockdown inhibited cellular invasion. (E) 3D invasion assay showed CSTI overexpression promoted cellular invasion, while CSTI knockdown inhibited cellular invasion. (F) WB analysis of E-cadherin, $\alpha$-catenin, fibronectin and vimentin expression after CSTI overexpression or knockdown, GAPDH was used as the loading control. Data were obtained from three independent experiments, and values are presented as the means \pm SEM. $* P<0.05$.

regulates EMT, WB analysis showed CST1 overexpression inhibited E-cadherin and $\alpha$-catenin expression, and promoted fibronectin and vimentin expression, while CST1 knockdown promoted E-cadherin and $\alpha$-catenin expression, and inhibited fibronectin and vimentin expression (Figure 2F). These results suggested that CST1 increased gastric cancer migration and invasion ability.

\section{CSTI Promoted Gastric Cancer Migration and Invasion Through Activating Wnt Pathway}

In order to determine the molecular mechanism of CST1 in gastric cancer progression, GSEA was used to determine which signaling pathway was regulated by CST1. High CST1 expression was positively correlated with high Wnt pathway activity (Figure 3A), Luciferase reporter assay suggested CST1 overexpression significantly upregulated Wnt pathway activity, while CST1 knockdown significantly downregulated Wnt pathway activity (Figure 3B). Western blot showed CST1 overexpression promoted the translocation of $\beta$-catenin into nuclear, while CST1 knockdown inhibited the translocation of $\beta$-catenin into nuclear (Figure 3C). We also analyzed the expression of MYC, CD44, SNAI1, RUNX2 and CCND1 after CST1 overexpression and knockdown. These genes all are associated with migration and invasion and the targets of Wnt pathway, Q-PCR analysis showed CST1 overexpression increased their expression, while CST1 knockdown inhibited their expression, suggesting CST1 increased Wnt pathway activity (Figure 3D). To determine whether CST1 promoted gastric cancer migration and invasion through activating the Wnt pathway, we inhibited TCF which is a coactivator of Wnt pathway using small interference RNA (siRNA) and Wnt pathway inhibitor iCRT3 in CST1 overexpressed cells and found inhibited Wnt pathway significantly inhibited gastric cancer cell migration and invasion (Figure 4A and B), suggesting CST1 
A

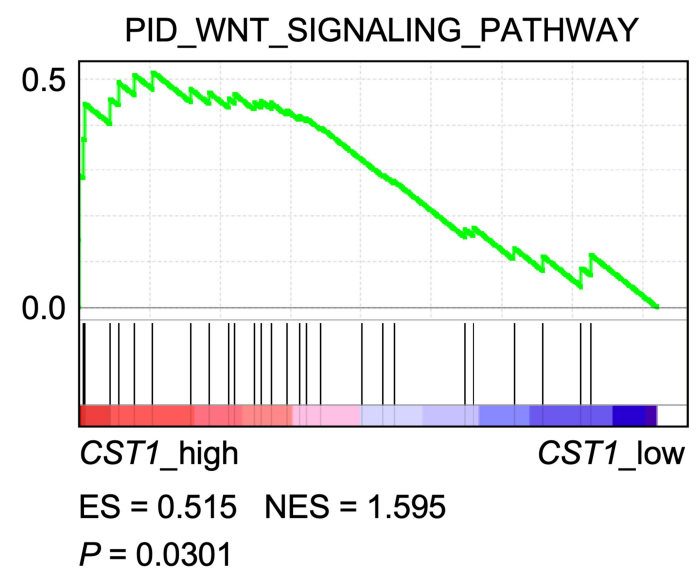

C

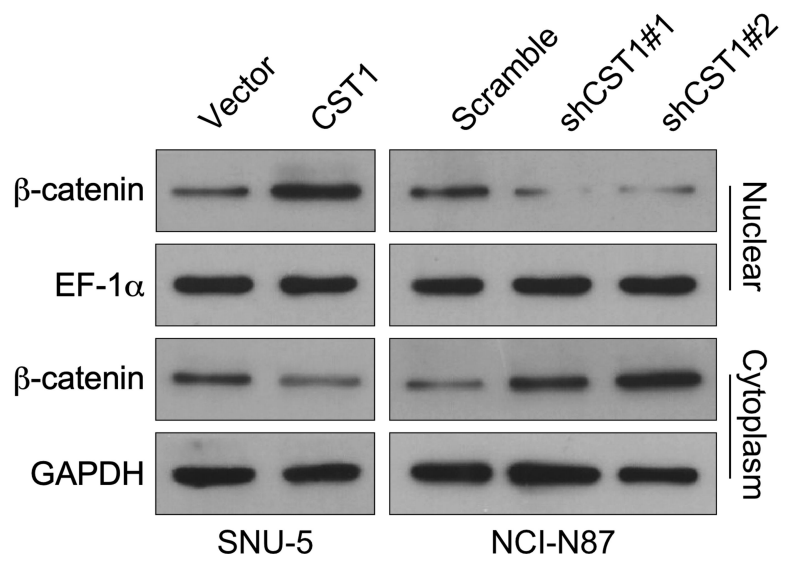

B

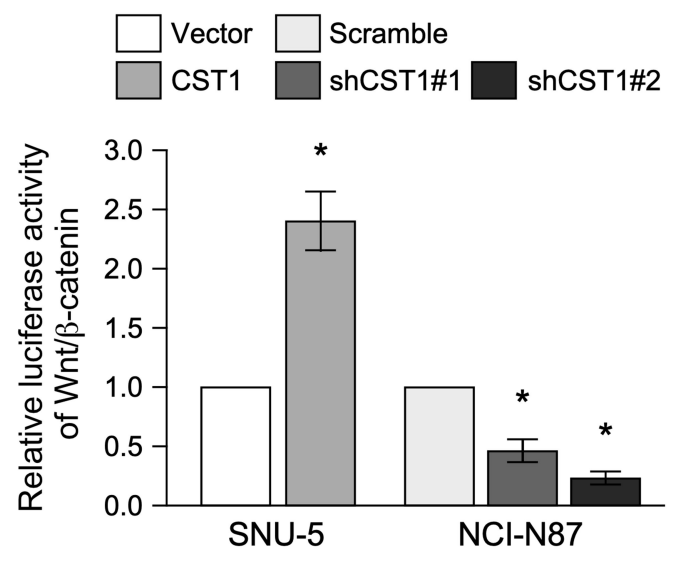

D

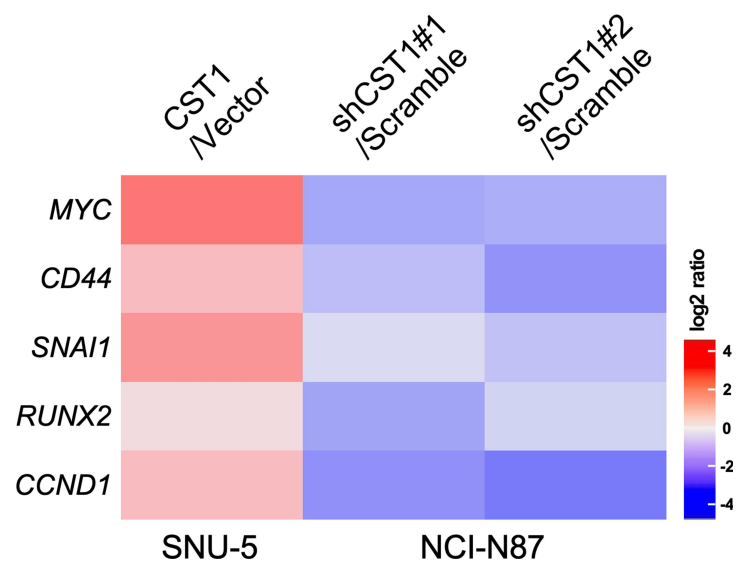

Figure 3 CSTI regulated Wnt pathway. (A) GSEA assay showed CSTI expression was positively correlated with Wnt signaling pathway activity. (B) Luciferase reporter assay showed CSTI overexpression increased Wnt pathway activity, while CSTI knockdown inhibited Wnt pathway activity. (C) Western blot analyzed $\beta$-catenin expression in nuclear and cytoplasm after CSTI overexpression or knockdown. (D) Heatmap showed Wnt pathway target genes expression after CST overexpression or knockdown. Data were obtained from three independent experiments, and values are presented as the means \pm SEM. $* P<0.05$.

increased gastric cancer cell migration and invasion through suppressing Wnt pathway activity.

\section{Discussion}

In the present study, CST1 was overexpressed in gastric cancer tissues and cells, and patients who had high CST1 levels had poor clinic outcome. CST1 promoted gastric cancer migration and invasion, mechanism analysis showed CST1 promoted gastric cancer migration and invasion through activating the Wnt pathway.

Previous studies show CST1 promotes gastric cancer proliferation, and we found CST1 promoted gastric cancer migration and invasion, ${ }^{11}$ further exploring the role of CST1 in gastric cancer progression. We found CST1 promoted gastric cancer migration and invasion through activating the Wnt pathway. The Wnt pathway is associated with gene mutations and often causes gastric cancer, for example, exon 3 mutation in $\beta$-catenin frequently occurrs in gastric cancer, while mutation of $\beta$-catenin can activate the Wnt pathway to promote gastric cancer progression. ${ }^{14}$ Wnt pathway has been shown to regulate gastric cancer progression. For example, Hsp90ab1 promoted gastric cancer invasion and metastasis through stabilizing LRP5 to activating Wnt pathway. ${ }^{15}$ ESRRG suppresses gastric cancer growth and tumorigenesis through inhibiting the binding of TCF4/LEF1 and CCDN1 promoter and inhibiting the Wnt pathway. ${ }^{16}$ Our results showed CST1 overexpression increased the nuclear translocation of $\beta$-catenin, promoting the Wnt pathway target genes expression, suggesting CST1 activated Wnt pathway. Further analysis showed double inhibition of CST1 and Wnt pathway promoted gastric cancer migration 
A

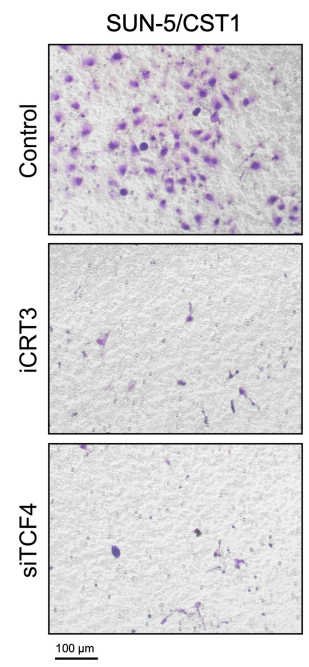

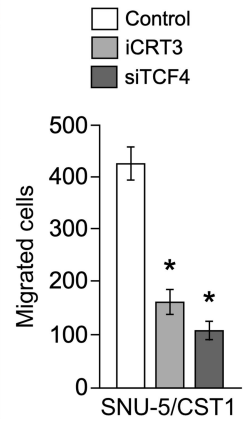

B

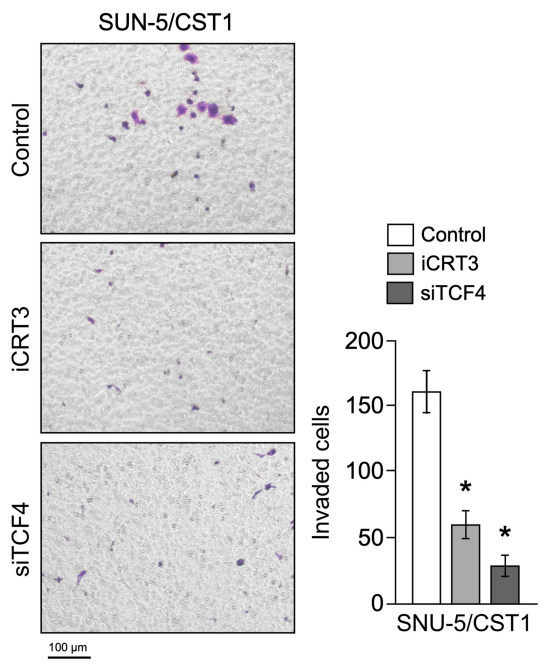

Figure 4 CSTI promoted gastric cancer migration and invasion through activating Wnt pathway. (A) Transwell chambers assay without Matrigel showed inhibition of Wnt pathway in CSTI overexpression cell inhibited cellular migration. (B) Transwell chambers assay with Matrigel showed inhibition of Wnt pathway in CSTI overexpression cell inhibited cellular invasion. Data were obtained from three independent experiments, and values are presented as the means \pm SEM. $* P<0.05$.

and invasion, confirming CST1 promoted gastric cancer migration and invasion through activating Wnt pathway, providing a new target for gastric cancer treatment.

\section{Abbreviations}

CST1, cystatin SN; NSCLC, non-small cell lung cancer; TCGA, The Cancer Genome Altas.

\section{Data Sharing Statement}

The data used to support the findings of this study are available from the corresponding author upon request.

\section{Funding}

This project is supported by New Medicine of USTC Joint Fund (No: WK9110000068).

\section{Disclosure}

The authors report no conflicts of interest in this work.

\section{References}

1. Chen C, Shi C, Huang X, et al. Molecular profiles and metastasis markers in Chinese patients with gastric carcinoma. Sci Rep. 2019;9 (1): 13995

2. Cristescu R, Lee J, Nebozhyn M, et al. Molecular analysis of gastric cancer identifies subtypes associated with distinct clinical outcomes. Nat Med. 2015;21(5):449-456.

3. Barrett AJ. The cystatins: a diverse superfamily of cysteine peptidase inhibitors. Biomed Biochim Acta. 1986;45(11-12):1363-1374.

4. Lah TT, Babnik J, Schiffmann E, Turk V, Skaleric U. Cysteine proteinases and inhibitors in inflammation: their role in periodontal disease. J Periodontol. 1993;64(5 Suppl):485-491.
5. Saitoh E, Kim HS, Smithies O, Maeda N. Human cysteine-proteinase inhibitors: nucleotide sequence analysis of three members of the cystatin gene family. Gene. 1987;61(3):329-338.

6. Jiang J, Liu HL, Liu ZH, Tan SW, Wu B. Identification of cystatin SN as a novel biomarker for pancreatic cancer. Tumour Biol. 2015;36 (5):3903-3910.

7. Jiang J, Liu HL, Tao L, et al. Let7d inhibits colorectal cancer cell proliferation through the CST1/p65 pathway. Int J Oncol. 2018;53 (2):781-790.

8. Yoneda $\mathrm{K}$, Iida $\mathrm{H}$, Endo $\mathrm{H}$, et al. Identification of Cystatin $\mathrm{SN}$ as a novel tumor marker for colorectal cancer. Int J Oncol. 2009;35 (1):33-40.

9. Cao X, Li Y, Luo RZ, et al. Expression of Cystatin SN significantly correlates with recurrence, metastasis, and survival duration in surgically resected non-small cell lung cancer patients. Sci Rep. 2015;5:8230.

10. Dai DN, Li Y, Chen B, et al. Elevated expression of CST1 promotes breast cancer progression and predicts a poor prognosis. $\mathrm{J} \mathrm{Mol} \mathrm{Med}$ (Berl). 2017;95(8):873-886.

11. Choi EH, Kim JT, Kim JH, et al. Upregulation of the cysteine protease inhibitor, cystatin $\mathrm{SN}$, contributes to cell proliferation and cathepsin inhibition in gastric cancer. Clin Chim Acta. 2009;406 (1-2):45-51.

12. Hnasko TS, Hnasko RM. The western blot. Methods Mol Biol. 2015;1318:87-96.

13. Cai J, Guan H, Fang L, et al. MicroRNA-374a activates Wnt/beta-catenin signaling to promote breast cancer metastasis. $J$ Clin Invest. 2013;123(2):566-579.

14. Clements WM, Wang J, Sarnaik A, et al. beta-Catenin mutation is a frequent cause of Wnt pathway activation in gastric cancer. Cancer Res. 2002;62(12):3503-3506.

15. Wang H, Deng G, Ai M, et al. Hsp90ab1 stabilizes LRP5 to promote epithelial-mesenchymal transition via activating of AKT and Wnt/ beta-catenin signaling pathways in gastric cancer progression. Oncogene. 2019;38(9):1489-1507.

16. Kang MH, Choi H, Oshima M, et al. Estrogen-related receptor gamma functions as a tumor suppressor in gastric cancer. Nat Commun. 2018;9(1):1920. 


\section{Publish your work in this journal}

Cancer Management and Research is an international, peer-reviewed open access journal focusing on cancer research and the optimal use of preventative and integrated treatment interventions to achieve improved outcomes, enhanced survival and quality of life for the cancer patient.

The manuscript management system is completely online and includes a very quick and fair peer-review system, which is all easy to use. Visit http://www.dovepress.com/testimonials.php to read real quotes from published authors. 